\title{
Translation and validation of the Sociocultural Attitudes Towards Appearance Questionnaire-4 in Iranian adolescents
}

\author{
Sohrab Amiri ${ }^{1}$, Amir Ghasemi Navab² \\ 'Urmia University, Urmia, Iran \\ ${ }^{2}$ Allameh Tabatabaei University, Tehran, Iran \\ Neuropsychiatria i Neuropsychologia 2018; 13, 2: 50-56
}

Address for correspondence:

Sohrab Amiri, PhD

Urmia University

Urmia, Iran

e-mail: amirysohrab@yahoo.com

\begin{abstract}
The goal of this research was to validate the Sociocultural Attitudes Towards Appearance Questionnaire- 4 (SATAQ-4) in the adolescent population. For this purpose, 662 male adolescents were selected, then SATAQ- 4 (Schaefer et al. 2015), Body Appreciation, Appearance Evaluation, Body Surveillance and Acceptance, and Action Questionnaire-II (AAQ-II) were distributed among them. The reliability was assessed by $\alpha$ coefficient, test-retest, and split-half coefficient, and the validity was assessed with other questionnaires to determine the psychometric properties of SATAQ-4. The confirmatory factor structure was assessed by confirmatory factor analysis. Factor analysis indicated that SATAQ-4 has five factors, and checking the validity of the inventory using Cronbach's $\alpha$, test-retest, and split-half coefficient reflected the stability of the scale; the criterion validity of the SATAQ- 4 with other questionnaires showed desirable discriminant and convergence validity. Overall, the findings indicated that SATAQ-4 has good psychometric properties in the adolescent population, and the tool can be used in studies in the general population. However, it seems that the SATAQ-4 subscales have low internal consistency in the Iranian adolescent population in comparison with the original version.
\end{abstract}

Key words: adolescent, body appearance, factor structure, validity, reliability.

\section{Introduction}

Body image includes complex attitudes, emotions, and behaviours towards appearance (Cash and Smolak 2011). Body image difficulties lie on a continuum from body image dissatisfaction to body image disturbance (Cash et al. 2004), which can be influenced by social and cultural dimensions.

An array of consequences are associated with body image disturbance (e.g. decreased selfesteem; Grossbard et al. 2009). Accordingly, researchers are trying to understand the aetiology of these disorders (Cash and Smolak 2011). Many researchers provide significant support for the influence of sociocultural factors on body image disturbances. Sociocultural models emphasise the influence of cultural factors on satisfaction and dissatisfaction of body appearance. The Tripartite Influence Model (Thompson et al. 1999) states that appearance-related pressures from three sociocultural factors: peers, family, and the media affect attitudes towards body image, leading to psychological problems. This model states that the effects of the sociocultural factors are mediated via two mechanisms: appearance-ideal internalisation and appearance comparison. The tripartite model has received adequate support in the Western samples (Shroff and Thompson 2006).

Adolescent girls are broadly affected by the media, which glorifies thinness and attractiveness. Therefore, girls are exposed to these messages and images that glorify a thin appearance ideal via different media (Thompson and Smolak 2001). The relationship between media pressure and dissatisfaction with body image in men has also been highlighted, and it has been shown that increased media pressure is associated with an increase in body dissatisfaction (Blond 2008; Knauss et al. 2007; Thompson and Cafri 2007). Exposure to thin media images and media pressure to attain a thin body leads to psychological problems including body concerns and eating disorders in girls (Harrison 2000). Peers are an- 
other influencing factor on body image during adolescence. Adolescents frequently engage in conversations about body appearance and attractiveness with their peers (Levine and Smolak 2004), and on the other hand, girls often receive critical comments about their body appearance from peers (Tantleff-Dunn and Gokee 2004), which predicts body image dissatisfaction. Interestingly, body image dissatisfaction shows similarities within friendship cliques (Paxton et al. 1999). Families also influence adolescents' body image (McCabe et al. 2003), criticism (Levine and Smolak 2004), and appearance-related attitudes (Vincent and McCabe 2000).

Research has shown that sociocultural factors have a strong influence on body image. However, more research is needed to investigate the interrelationships between sociocultural factors and attitudes towards body image in adolescents in Eastern culture. Moreover, validation of common measures that assess sociocultural factors allow researchers to examine sociocultural factors of body image disturbances within adolescent samples.

The Sociocultural Attitudes Towards Appearance Questionnaire (SATAQ) was developed to evaluate sociocultural factors of body image difficulties (Heinberg et al. 1995). This scale has been reviewed and revised several times (Thompson et al. 1999). The SATAQ-3 focused on media effects (Thompson et al. 2004). This scale was validated in several countries, including the United States (Thompson et al. 2004; Rousseau et al. 2010; Llorente et al. 2013).

Although the validity of SATAQ-3 is supported, the scale has its limits (Schaefer $e t$ al. 2015). Firstly, solely assesses the effects of the popular media. Secondly, the items do not obviously assess ideal internalisation. To address these limitations, Schaefer et al. (2015) revised SATAQ-3 and developed SATAQ-4. A five-factor model with five subscales: 1) Thin/Low Body Fat, for assessing the extent that the person endorses a thin body as an ideal; 2) Muscular/Athletic, for assessing the extent that the person endorses an athletic body as an ideal; 3) Family Pressures: for assessing the extent that the person feels pressure from their family in order to achieve a certain appearance; 4) Peer Pressures: for assessing the extent that the person feels pressure from their peers to achieve a certain appearance; and 5) Media Pressures: for assessing the extent that the person feels pressure from the media to achieve a certain appearance.

SATAQ-4 has already been validated cross-culturally in different cultural samples (e.g. the United States, England, and Italy [Schaefer et al. 2015]). However, the psychometric properties of the measure have not yet been examined in Eastern and Iranian culture. Accordingly, the present study has two objectives: 1) investigating the factor structure of the SATAQ- 4 and 2) examining the internal consistency and validity of the SATAQ- 4 in Iranian adolescents as an Eastern culture.

\section{Material and methods}

\section{Participants and procedure}

The study participants comprised 662 male students who were selected through multi-stage cluster sampling among students (public and private schools). Inclusion criteria were being between 12 and 18 years old, and all participants reported that they had not been diagnosed with any medical condition by a doctor. Participants with a psychotic disorder, problems with substances, acute suicidality, insufficient language skills, or severe cognitive impairment were excluded.

Participants completed a series of questionnaires following an informed consent procedure and were provided with debriefing information on the purpose of the study and given a list of community counselling agencies at the end of the survey. Of the total participants, 484 were at the first year of high school (73.1\%), and 178 were in the second year of high school $(26.9 \%)$, 390 participants were male (58.9\%), and 272 were female (41.1\%). Respectively, the mean students' ages and standard deviation were 14.25 and 0.87 in males, 14.21 and 0.95 in females, respectively.

\section{Measures \\ Sociocultural attitudes towards Appearance Questionnaire-4}

The SATAQ-4 is a 22-item questionnaire with five subscales: Thin/Low Body Fat, Muscular/Athletic, Family Pressures, Peers Pressures, and Media Pressures. Responses are made on a Likert scale. This scale has good psychometric properties in the United States, Italy, England, and Australia (Schaefer et al. 2015). In order to provide the Persian version of the SATAQ-4, the researchers and two experts in English language translated the questionnaire into fluent Persian, and then the translated text was edited. At the next step, the translated version was turned into English language again by the researcher and the two language experts, and after that 
the two versions were reconciled. To examine the face validity, the questionnaire was given to two psychologists. After reconciling the two translated and main versions and eliminating the mistakes, the questionnaire was conducted on 40 students who were similar to the final population, and after receiving the essential feedback on the content of the items and fixing the raised weaknesses, the final version of the questionnaire was prepared for use.

\section{Body Appreciation}

The 19 items in the BAS-2 item pool were administered. Responses are made on a Likert scale (Avalos et al. 2005).

\section{Appearance Evaluation}

The seven-item Appearance Evaluation subscale of the Multidimensional Body Self-Relations Questionnaire (MBSRQ; Brown et al. 1990) assessed participants' perceived self-attractiveness. Responses are made on a Likert scale. Higher values show a more favourable appearance evaluation. Among college women, the Appearance Evaluation subscale was found to have good psychometric properties (Avalos et al. 2005).

\section{Body Surveillance}

Body Surveillance (McKinley and Hyde 1996) measured the tendency of habitually monitoring one's appearance. Responses are made on a Likert scale. The Body Surveillance subscale has good psychometric properties (McKinley and Hyde 1996).

\section{Acceptance and Action Questionnaire-II}

The AAQ-II comprises 10 items on a Likert scale. The AAQ-II can be scored in the direction of psychological flexibility or psychological inflexibility. The AAQ-II was normed using diverse samples. This scale has good psychometric properties with highly favourable validity and internal consistency: 0.81 to 0.87 (Bond et al. 2011).

\section{Data analytic strategy}

SPSS version 22 (SPSS IBM, New York) and LISREL software (Jöreskog and Sörbom 2006) were used to perform statistical analyses. Bivariate correlations, Cronbach's $\alpha$, split-half and test-retest coefficients, and confirmatory factor analysis were calculated to examine the convergence among body appreciation, appear- ance evaluation, body surveillance, and AAQ-II with sociocultural attitudes towards appearance variables, internal consistency, and factor structure of SATAQ-4. In order to investigate the fit of the three-factor structure of the SATAQ-4 (Schaefer et al. 2015), confirmatory factor analysis by maximum likelihood method and LISREL software were used (Jöreskog and Sörbom 2006). To investigate the reliability of the SATAQ-4, Cronbach's $\alpha$, split-half, and test-retest coefficients were calculated.

\section{Results}

\section{Factor analysis}

The diagram of conformity factor analysis path with path coefficients are shown in Figure 1, the $\mathrm{T}$ index is also presented in Table 1 , and the fit indices of the model are presented in Table 2.

The closer the scores of normed fit index (NFI), relative fit index (RFI), and goodness of fit index (GFI) are to 1, more they indicate the pattern's desired fit. In the present study, the most valid fit scores have been used in order to assess the model's fit. Standardised root mean square residual $(\mathrm{SRMR})<0.08$ shows a desired fit, and SRMR $<0.10$ indicates an acceptable fit, and the model fails when SRMR > 0.10 (Hu and Bentler 1999). The index with the value of RMSEA $\leq 0.8$ shows the model is good, and when the RMSEA is between 0.10 and 0.08 , the model is acceptable. Eventually, CFI $\geq 0.95$ states a good fit of the model ( $\mathrm{Hu}$ and Bentler 1999). Since the $\chi^{2}$ statistic is sensitive to the sample size, to assess the overall fit of the model, the amount of $\chi^{2}$ is calculated along with the $\mathrm{df}\left(\chi^{2} / \mathrm{df}\right)$. The $\chi^{2} / \mathrm{df}<2$ represents the model's good fit, and when $\chi^{2} / \mathrm{df}$ is around 3 the model is acceptable. The amount of $\chi^{2} / \mathrm{df}$ was 3.01 , which is around 3 , so the model's fit is acceptable. In addition, the $\mathrm{SRMR}=0.08$ fit index showed the three-factor model's acceptable fit, and the RMSEA = 0.06 showed the good fit of the model (Brown 2006; Schermelleh-Engel et al. 2003; Hair et al. 2009; Hu and Bentler 1999; Tabachnick and Fidell 2007). Although the CFI index was not desirable, the total of the fitness indicators should be interpreted together, and the model was supported by total fitness indicators.

\section{Validity and reliability}

The 22-question scale's Cronbach's coefficient was 0.82 , which showed that the scale has a good internal consistency. Cronbach's coefficient was 0.82 for Thin/Low Body Fat, Muscular/Athletic, 


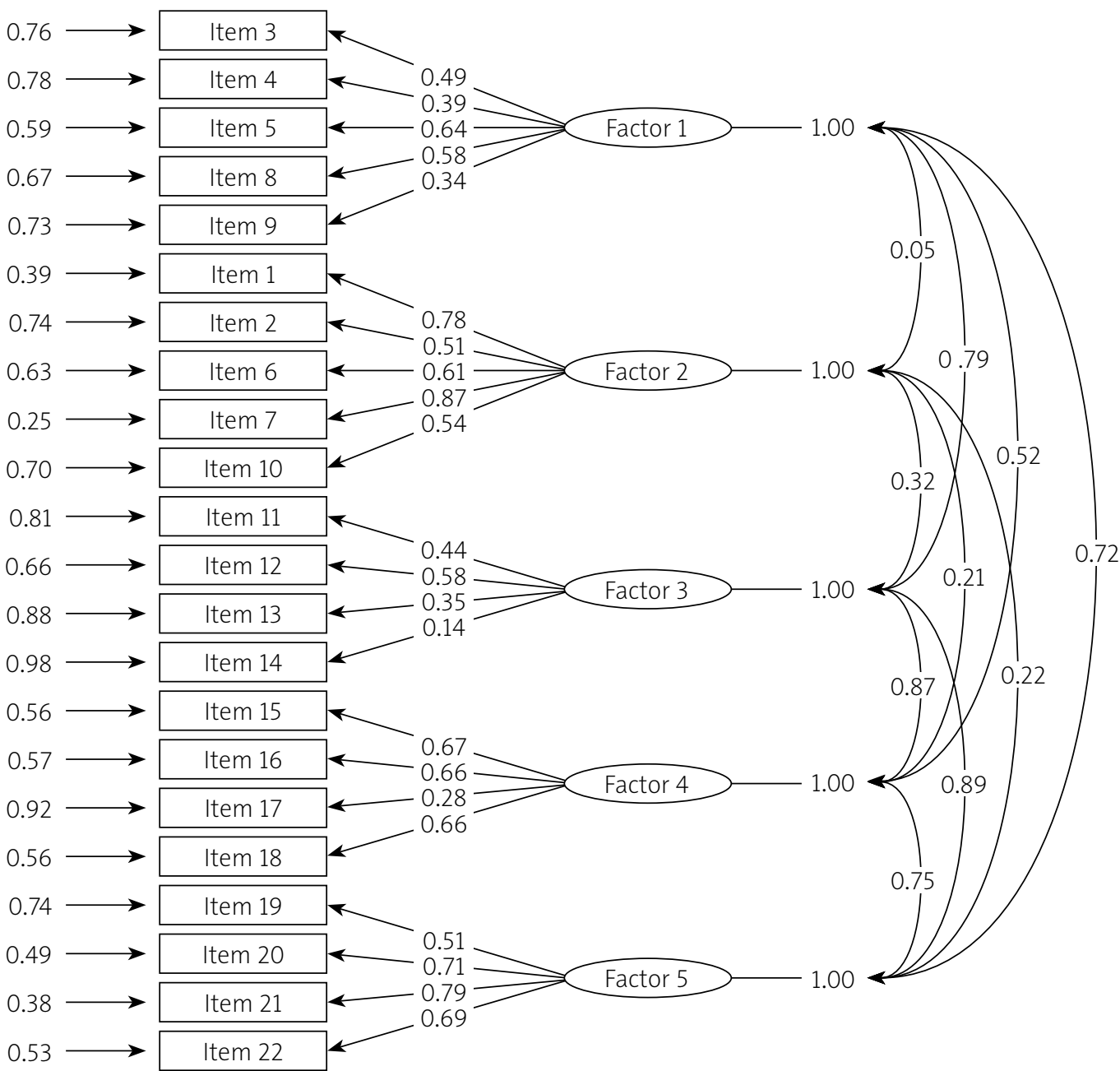

Fig. 1. Diagram of confirmatory factor analysis and path coefficients of Sociocultural Attitudes Towards Appearance Questionnaire-4

Family, Peers, and Media scales were 0.80, 0.79, $0.76,0.64$, and 0.76 , respectively. The split-half coefficient also indicated the scale and its subscale's high reliability. Ninety participants (one missing) answered the questionnaire questions again four weeks later to calculate the test-retest coefficient, and the obtained scores' correlation coefficients were calculated after the conduction of the three tests. The results of the Cronbach's $\alpha$, the split-half, and test-retest coefficients are presented in Table 3.

Table 3 shows that Cronbach's $\alpha$ coefficients were satisfactory, and the data of all the testretest and split-half s coefficients were significant. Therefore, it can be concluded that the SATAQ-4 has desirable internal consistency in the adolescent population. However, the SATAQ-4 subscales have low internal consistency in the Eastern adolescent population in comparison with the original version.

The SATAQ-4 (Schaefer $e$ t al. 2015) validity in the adolescent population was examined in three ways: the construct validity (conducting simultaneously with Body Appreciation [Avalos et al. 2005], Appearance Evaluation [Brown et al. 1990], Body Surveillance [McKinley and Hyde 1996], and AAQ-II [Bond et al. 2011]) and the correlation between subscales. The results are shown in Table 4.

The correlation coefficients between the subscales with Body Appreciation (Avalos et al. 2005), Appearance Evaluation (Brown et al. 1990), Body Surveillance (McKinley and Hyde 1996), and AAQ-II (Bond et al. 2011), indicate the construct validity of the SATAQ-4 (Schaefer et al. 2015) in the adolescent population.

\section{Conclusions}

The present study was performed to evaluate and validate the SATAQ- 4 in the adolescent population. The SATAQ-4 factor analysis showed that the five-factor solution has a good fit. This finding is consistent with the studies 


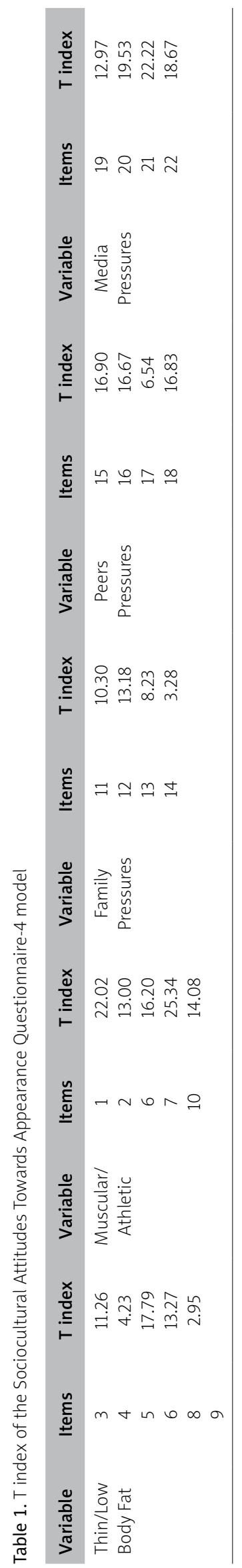

that examine the scale SATAQ-4 factor structure (Schaefer $e t$ al. 2015). The findings also indicated that the five-factor solution has desirable internal reliability, similar to that of the factor structure of the original questionnaire. Studying the SATAQ-4's factor structure and patterns of factor loadings, using confirmatory factor analysis represented the same results as the original version (Schaefer et al. 2015) and a Japanese female sample (Yamamiya et al. 2016) by five-factor solution. All the factor loadings were higher than 0.4 .

Investigating the SATAQ-4's reliability, using $\alpha$ coefficients, test-retest coefficients and splithalf showed the scale's appropriate reliability. The subscale's $\alpha$ coefficients were, respectively, $0.80,0.79,0.76,0.64$, and 0.76 , and the amplitude of test-retest and split-half s coefficients suggested the SATAQ-4's suitable reliability to measure the sociocultural attitudes towards appearance in the adolescent population. The findings are consistent with the study of Schaefer et al. (2015), who designed the original measure of SATAQ- 4 and the Japanese version (Yamamiya et al. 2016). The results of Cronbach's $\alpha$ coefficients in the adolescent population were different and lower than the two above study; however, the results indicate acceptable internal consistency. Perhaps the reason for this difference in Cronbach's $\alpha$ rate is the issue of cultural differences that affects body image, because the above studies have been carried out in developed countries where the influence of the media is more intense than the culture in which the current study was conducted. Perhaps with some changes in the questionnaire, a better questionnaire can be made for future studies, especially in clinical samples. For example, deleting some items that have a very low load factor, as well as items that are not very useful in Iranian culture, can be useful.

The validity of the SATAQ- 4 with Body Appreciation (Avalos et al. 2005), Appearance Evaluation (Brown et al. 1990), Body Surveillance (McKinley and Hyde 1996), and AAQ-II (Bond et al. 2011) indicates the validity of the SATAQ-4 (Schaefer et al. 2015), and this correlation was significant, and it shows that SATAQ-4 can be a good scale for measuring aspects of SATAQ-4. This result demonstrated the divergent validity of the SATAQ- 4 . The SATAQ-4 psychometric properties of the present study in the adolescent population were respectively consistent with the studies done in the original version (Schaefer et al. 2015). According to what was said, the lack of a concise yet valid and 
Table 2. The fit indices of the Sociocultural Attitudes Towards Appearance Questionnaire-4 model

\begin{tabular}{ccccccccccccc} 
Index & $\chi^{2}$ & $\mathrm{df}$ & $p$-value & RMSEA & SRMR & NFI & NNFI & RFI & IFI & GFI & AGFI & CFI \\
Value & 599.45 & 199 & $>0.05$ & 0.06 & 0.08 & 0.95 & 0.91 & 0.90 & 0.97 & 0.98 & 0.89 & 0.67 \\
\hline
\end{tabular}

RMSEA - root mean square error of approximation, SRMR - standardised root mean square residual, NFI-normed fit index, NNFI-non-normed fit index, RFI - relative fit index, IFI - incremental fit index, GFI - goodness of fit index, AGFI-adjusted goodness of fit index, CFI - comparative fit index

Table 3. The mean, standard deviation, $\alpha$, test-retest, and split-half coefficient of the Sociocultural Attitudes Towards Appearance Questionnaire-4

\begin{tabular}{lccccc} 
Variable & Items & M/SD & $\begin{array}{c}\text { Cronbach's } \alpha \\
(n=662)\end{array}$ & $\begin{array}{c}\text { Test-retest } \\
(n=89)\end{array}$ & $\begin{array}{c}\text { Split-half } \\
\text { coefficient } \\
(n=662)\end{array}$ \\
\hline Thin/Low Body Fat & $3-5,8,9$ & $11.99(3.40)$ & 0.80 & $0.58^{* *}$ & 0.68 \\
\hline Muscular/Athletic & $1,2,6,7,10$ & $16.79(4.71)$ & 0.79 & 0.87 \\
\hline Family Pressures & $11-14$ & $10.54(3.02)$ & 0.76 & $0.49^{* *}$ & $0.52^{* *}$ \\
\hline Peers Pressures & $15-18$ & $8.45(3.42)$ & 0.64 & $0.67^{* *}$ & 0.75 \\
\hline Media Pressures & $19-22$ & $8.90(3.99)$ & 0.76 & 0.79 \\
\hline$* * 0.01$ & & &
\end{tabular}

Table 4. Correlations between the subscales of Sociocultural Attitudes Towards Appearance Questionnaire-4 and convergent measures

\begin{tabular}{|c|c|c|c|c|c|c|c|c|c|}
\hline Variable & 1 & 2 & 3 & 4 & 5 & $\begin{array}{l}\text { Body Sur- } \\
\text { veillance }\end{array}$ & $\begin{array}{c}\text { Appear- } \\
\text { ance Eval- } \\
\text { uation }\end{array}$ & $\begin{array}{c}\text { Body } \\
\text { Appreciation }\end{array}$ & AAQ-II \\
\hline Thin/Low Body Fat & 1 & & & & & $0.16^{*}$ & $-0.11^{\star}$ & -0.09 & $0.13^{\star}$ \\
\hline Muscular/Athletic & $0.26^{* *}$ & 1 & & & & $0.19^{\star \star}$ & $-0.17^{\star *}$ & $-0.14^{\star}$ & $0.19^{* *}$ \\
\hline Family & $0.20^{\star \star}$ & $0.34^{\star *}$ & 1 & & & $0.23^{\star \star}$ & $-0.23^{\star *}$ & $-0.012^{*}$ & $0.28^{* *}$ \\
\hline Peers & $0.33^{\star *}$ & $0.28^{* *}$ & $0.48^{* *}$ & 1 & & $0.18^{* *}$ & $-0.18^{\star \star}$ & $-0.19^{\star *}$ & $0.021^{* *}$ \\
\hline Media & $0.46^{\star *}$ & $0.20^{\star *}$ & $0.50^{\star \star}$ & $0.55^{\star *}$ & 1 & $0.14^{*}$ & $-0.15^{\star}$ & $-0.18^{\star *}$ & $0.24^{* *}$ \\
\hline
\end{tabular}

useful tool to assess the SATAQ-4 is the weakness of the research in this field. As a result, it seems that, regardless of language and culture, and considering the pattern of factor loadings, SATAQ-4 is a useful tool in measuring the fundamental structures related to sociocultural attitudes towards appearance. But it seems that in clinical samples greater care should be taken. In total, the psychometric properties of SATAQ-4 are applicable broadly and have the capacity to measure sociocultural attitudes towards appearance associated with a variety of disorders, and it can also be used in clinical and normal populations.

In total, the reliability, validity, and confirmatory factor analysis demonstrated the desirable psychometric characteristics of the SATAQ-4, and the present findings are consistent with those of the original version (Schaefer et al. 2015). The SATAQ-4 in the adolescent population samples showed that it is a valid tool for assessing sociocultural attitudes towards appearance. The calculated indices to evaluate the fit of the SATAQ-4's model suggested that SRMR, RMSEA, and $\chi^{2} / \mathrm{df}$ indices, as the most valid fit indices (Brown 2006; Schermelleh-Engel et al. 2003; Hair et al. 2009; Hu and Bentler 1999; Tabachnick and Fidell 2007), support the five-factor model's fit. So, according to what was said, the present study was conducted among adolescent population samples, and because it did not cover the all other groups, the results should be treated with caution in generalising. It is also suggested that future studies examine the SATAQ-4's validity by using other psychological methods in clinical and other groups. The results of the present study indicates that the SATAQ-4 has acceptable validity and reliability in adolescents. In addition, the questionnaire's factor structure was compliant with the design- 
ers' theory, and the three produced factors of the confirmatory factor analysis were consistent with the original version of the SATAQ-4 (Schaefer et al. 2015).

\section{References}

1. Avalos LC, Tylka TL, Wood-Barcalow N. The Body Appreciation Scale: Development and psychometric evaluation. Body Image 2005; 2: 285-297.

2. Blond A. Impacts of exposure to images of ideal bodies on male body dissatisfaction: A review. Body Image 2008: 5; 244-250.

3. Bond FW, Hayes SC, Baer RA, et al. Preliminary psychometric properties of the acceptance and action questionnaire-II: a revised measure of psychological inflexibility and experiential avoidance. Behav Ther 2011; 42: 676-688.

4. Brown TA. Confirmatory factor analysis for applied research. Guilford Press, New York 2006.

5. Brown TA, Cash TF, Mikulka PJ. Attitudinal body image assessment: Factor analysis of the Body-Self Relations Questionnaire. J Pers Assess 1990; 54: 213-230.

6. Cash TF, Smolak L (Eds.). Body image: A handbook of science, practice, and prevention ( $2^{\text {nd }}$ ed.). Guilford Press, New York 2011.

7. Cash TF, Phillips KA, Santos MT, Hrabosky JI. Measuring "negative body image": validation of the body image disturbance questionnaire in a nonclinical population. Body Image 2004; 1: 363-372.

8. Grossbard JR, Lee CM, Neighbors C, Larimer ME. Body image concerns and contingent self-esteem in male and female college students. Sex Roles 2009; 60: 198-207.

9. Hair JF, Black WC, Babin BJ, Anderson RE. Multivariate data analysis. Prentice Hall, 2009.

10. Harrison K. The body electric: Thin-ideal media and eating disorders in adolescents. J Commun 2000; 50: 119-143.

11. Heinberg LJ, Thompson JK, Stormer S. Development and validation of the Sociocultural Attitudes Towards Appearance Questionnaire. Int J Eat Disord 1995; 17: 81-89.

12. Hu L-T, Bentler PM. Cutoff criteria for fit indexes in covariance structure analysis: Conventional criteria versus new alternatives. Struct Equ Modeling 1999; 6: 1-55.

13. Jöreskog KG, Sörbom D. LISREL 8.80 for Windows [Computer Software]. Scientific Software International, Inc. Lincolnwood, IL 2006.

14. Knauss C, Paxton SJ, Alsaker FD. Relationships amongst body dissatisfaction, internalization of the media body ideal and perceived pressure from media in adolescent girls and boys. Body Image 2007; 4: 353-360.

15. Levine MP, Smolak L. Body image development in adolescence. In: Cash TF, Smolak L. (Eds.). Body image: A handbook of theory, research, and clinical practice. Guilford Press, New York 2004: 74-82.

16. Llorente E, Warren CS, de Eulate LP, Gleaves DH. A Spanish version of the Sociocultural Attitudes Towards Appearance Questionnaire-3 (SATAQ-3): Translation and psychiatric evaluation. J Clin Psychol 2013; 69: 240-251.

17. McCabe MP, Ricciardelli LA, Ridge D. Who thinks I need a perfect body? Perceptions and internal dialogue among adolescents about their bodies. Sex Roles 2003; 55: 409-419.
18. McKinley NM, Hyde JS. The Objectified Body Conscious ness Scale: Development and validation. Psychol Women Q 1996; 20: 181-215.

19. Paxton SJ, Schutz HK, Wertheim EH, Muir SL. Friendship clique and peer influences on body image concerns, dietary restraint, extreme weight-loss behaviors, and binge eating in adolescent girls. J Abnorm Psychol 1999; 108: 255-266.

20. Rousseau A, Valls M, Chabrol H. Validation of the French version of the Sociocultural Attitudes Towards Appearance Scale-3 (SATAQ-3). L'Encéphale 2010; 36: 270-276.

21. Schaefer LM, Burke, NL, Thompson JK, et al. Development and validation of the Sociocultural Attitudes Towards Appearance Questionnaire-4 (SATAQ-4). Psychol Assess 2015; 27: 54-67.

22. Schermelleh-Engel K, Moosbrugger H, Müller H. Evaluating the fit of structural equation models: Tests of significance and descriptive goodness of fit measures. Psychol Res 2003; 8: 23-74.

23. Shroff H, Thompson JK. Peer influences, body-image dissatisfaction, dysfunction and self-esteem in adolescent girls eating. J Health Psychol 2006; 11: 533-551.

24. Tabachnick BG, Fidell LS. Using multivariate statistics. Allyn and Bacon, Boston 2007.

25. Tantleff-Dunn S, Gokee JL. Interpersonal influences on body image development. In: Cash TF, Smolak L (Eds.) Body image: A handbook of theory, research, and clinical practice. Guilford Press, New York 2004: 108-116.

26. Thompson JK, Smolak L. (Eds.). Body image, eating disorders, and obesity in youth: Assessment, prevention, and treatment. American Psychological Association, Washington, DC 2001.

27. Thompson JK, Heinberg LJ, Altabe MN, Tantleff-Dunn S. Exacting beauty: Theory, assessment and treatment of body image disturbance. American Psychological Association, Washington, DC 1999.

28. Thompson JK, Cafri G (Eds.). The muscular ideal: Psychological, social, and medical perspectives. American Psychological Association, Washington, DC 2007.

29. Thompson JK, van den Berg P, Roehrig M, et al. The Sociocultural Attitudes Towards Appearance Scale-3 (SATAQ-3): Development and validation. Int J Eat Disord 2004; 35: 293-304

30. Vincent MA, McCabe MP. Gender differences among adolescents in family, and peer influences on body dissatisfaction, weight loss, and binge eating behaviors. J Youth Adolescence 2000; 29: 205-221.

31. Yamamiya Y, Shimai S, Schaefer LM, et al. Psychometric properties and validation of the Sociocultural Attitudes towards Appearance Questionnaire-4 (SATAQ-4) with a sample of Japanese adolescent girls. Body Image 2016; 19: 89-97. 